Fetal Diagnosis and Therapy

\title{
Outcome of Multifetal Pregnancy Reduction in Women with a Dichorionic Triamniotic Triplet Pregnancy to a Singleton Pregnancy: A Retrospective Nationwide Cohort Study
}

\author{
L. van de Mheen ${ }^{a}$ S.M.P. Everwijn ${ }^{b}$ M.C. Haak ${ }^{b}$ G.T.R. Manten ${ }^{c}$ \\ H.A. Zondervan ${ }^{d}$ M.F.C.M. Knapen ${ }^{j}$ M.A.J. Engels ${ }^{a}$ J.J.H.M. Erwich ${ }^{\mathrm{e}}$ \\ A.B. Coumans ${ }^{f} \quad$ J.M.G. van Vugt ${ }^{g} \quad$ C.M. Bilardo ${ }^{\text {e }}$ M.G. van Pampus ${ }^{h}$ \\ C.J.M. de Groot ${ }^{\mathrm{a}}$ B.W.J. Mol ${ }^{\mathrm{k}}$ E. Pajkrt ${ }^{\mathrm{i}}$
}

Departments of Obstetrics and Gynecology, ${ }^{a}$ VU Medical Center, Amsterdam, beiden University Medical Center, Leiden, ' University Medical Center, Utrecht, ${ }^{d}$ Rijnstate Hospital, Arnhem, ${ }^{e}$ University Medical Center, University of Groningen, Groningen, ${ }^{\mathrm{f}}$ Maastricht University Medical Center, Maastricht, ${ }^{9}$ Radboud University Medical Center, Nijmegen, h Onze Lieve Vrouwe Gasthuis, Amsterdam, and 'Academic Medical Center, Amsterdam, and ${ }^{\text {j}}$ Department of Obstetrics and Prenatal Medicine, Erasmus Medical Center, Rotterdam, The Netherlands; ${ }^{\mathrm{k}}$ The Robinson Institute, School of Reproductive Health and Paediatrics, University of Adelaide, Adelaide, S.A., Australia

\section{Key Words}

Multifetal pregnancy reduction - Triplet pregnancy ·

Monochorionicity · Pregnancy outcome

\begin{abstract}
Objective: To study the pregnancy outcomes of women with a dichorionic triamniotic triplet pregnancy that was reduced to a singleton pregnancy and to review the literature. Methods: We performed a nationwide retrospective cohort study. We compared time to delivery and perinatal mortality in dichorionic triplet pregnancies reduced to singletons with ongoing dichorionic triplet pregnancies and primary singleton pregnancies. Additionally, we reviewed the literature on the subject. Results: We studied 46 women with a reduced dichorionic triplet pregnancy and 42 women with an ongoing dichorionic triplet pregnancy. Median gestational age at delivery was 38.7 vs. 32.8 weeks, respectively ( $p<0.001$ ). Deliv-
\end{abstract}

ery <24 weeks occurred in 9 (19.6\%) women with a reduced triplet pregnancy and 4 (9.5\%) with an ongoing triplet pregnancy $(p=0.19)$. Perinatal survival rates between the reduced group and the ongoing triplet group were not significantly different. Conclusion: Multifetal pregnancy reduction in women with a dichorionic triplet pregnancy to a singleton pregnancy prolongs median gestational age at birth. No statistically significant association was found with miscarriage and perinatal survival rates.

(c) 2015 S. Karger AG, Basel

\section{Introduction}

Due to restrictions in the number of embryos transferred in women undergoing assisted reproductive technology (ART), the number of (higher-order) multiple pregnancies has declined in the past years [1]. However,

\section{KARGER}

E-Mail karger@karger.com www.karger.com/fdt (c) 2015 S. Karger AG, Basel

$1015-3837 / 15 / 0402-0094 \$ 39.50 / 0$
Lidewij van de Mheen, MD

Department of Obstetrics and Gynecology, VU Medical Center PO Box 7075

NL-1007 MB Amsterdam (The Netherlands)

E-Mail l.vandemheen1@vumc.nl 
also after a single or double embryo transfer, a triplet or higher-order multiple pregnancy can occur, often containing a monozygotic twin. This could be caused by a less stable zona pellucida after intracytoplasmic sperm injection or after embryo transfer in a blastocyst stage $[2,3]$. Depending on the time of splitting of the embryo after conception, a monozygotic twin will have either a monochorionic or a dichorionic placenta [4]. Independent of this, ovulation induction and intrauterine insemination with ovarian hyperstimulation bear a risk of triplet pregnancy. Finally, increasing maternal age contributes directly to an increase in multiple pregnancies, including triplet pregnancies.

In the event of a triplet pregnancy, a woman can opt for multifetal pregnancy reduction (MFPR). In a previous study on MFPR in women with a trichorionic triplet pregnancy, we showed that MFPR to a twin can prolong pregnancy by 3 weeks, but with limited impact on neonatal survival [5]. In case of a triplet pregnancy with a monochorionic twin and a singleton alongside, the woman is exposed to the risks of both a triplet pregnancy, such as (very) preterm birth (PTB) and selective growth restriction, as well as the risks of a monochorionic multiple pregnancy, such as twin-to-twin transfusion syndrome (TTTS), twin anaemia-polycythaemia sequence (TAPS) and selective intrauterine growth restriction [6]. In women opting for MFPR in this situation, there is debate whether to reduce the monochorionic pair and keep the singleton, to reduce the singleton and keep the monochorionic twin, or to reduce one of the monochorionic multiples and continue the pregnancy as a dichorionic twin.

Some small studies showed that it is safer to reduce the monochorionic twin and retain a singleton pregnancy [7-10]. The aim of this study was to evaluate the outcome of MFPR in women with a dichorionic triamniotic triplet pregnancy to a singleton pregnancy by reducing the monochorionic pair. Additionally, we performed a review of the literature.

\section{Methods}

In this retrospective study we included all women with a dichorionic triamniotic triplet pregnancy undergoing fetal reduction to a singleton pregnancy in the Netherlands over the period 20002010. In seven centres data were available until 2013 (Academic Medical Center Amsterdam, VU Medical Center Amsterdam, Leiden University Medical Center, Maastricht University Medical Center, Radboud University Medical Center Nijmegen, Rijnstate Hospital Arnhem, and University Medical Center Groningen).

In all centres, the procedure was performed transabdominally with or without local anaesthetics by using a $20 \mathrm{G}$ or a $22 \mathrm{G}$ needle and injecting potassium chloride intracardially or intrathoracically to one or both fetuses of the monochorionic pair, based on the operators' discretion. Women were identified by searching the ultrasound, obstetric and fetal databases in all ten fetal medicine units performing MFPR. No preselection was made for indication of reduction.

The course of pregnancy in women with a reduced dichorionic triplet pregnancy was compared to that of women with an ongoing dichorionic triplet pregnancy as well as that of women with a primary singleton pregnancy. Data regarding ongoing triplet pregnancies were collected from ultrasound and obstetric databases in two tertiary hospitals (Academic Medical Center Amsterdam and VU Medical Center Amsterdam) and nine secondary hospitals (Onze Lieve Vrouwe Gasthuis Amsterdam, Zaans Medisch Centrum Zaandam, Spaarne Ziekenhuis Hoofddorp, Kennemer Gasthuis Haarlem, Rode Kruis Ziekenhuis Beverwijk, Medisch Centrum Alkmaar, Gemini Ziekenhuis Den Helder, Flevo Ziekenhuis Almere, and Ter Gooi Ziekenhuis Blaricum). Both tertiary hospitals serve a large geographical area in the Dutch provinces NoordHolland and Flevoland, including all nine above-mentioned secondary hospitals. All dichorionic triplet pregnancies in the period 2000-2012 with at least one ultrasound performed between $8^{0 / 7}$ and $14^{6 / 7}$ weeks gestation in which three living fetuses were identified and fetal reduction was not performed were included in the control group of ongoing triplet pregnancies. For our control group, we only included women with a known date of delivery.

The control group of primary singleton pregnancies was selected from women with a vital singleton pregnancy who attended antenatal care at a secondary hospital (Onze Lieve Vrouwe Gasthuis Amsterdam) prior to 16 weeks' gestation without a medical reason for secondary antenatal care and with a known date of delivery. Some women had two or more singleton pregnancies in the study period that met all inclusion criteria; in that case we used the first pregnancy only.

Data were collected retrospectively by reviewing maternal and, where appropriate, neonatal and paediatric medical records. Maternal and fetal characteristics including demographic data, gestational age (GA) at time of reduction, ultrasound findings including fetal measurements, chorionicity and pregnancy outcome were collected. Pregnancy dating was based on early ultrasound measurements. Chorionicity was determined at a first-trimester ultrasound scan or at post-delivery histological analysis by a pathologist. Operator and procedure characteristics were collected, including the number of operators and the number of procedures per operator.

Pregnancy outcome was based on GA at delivery, neonatal birth weight and perinatal death. All cases of fetal demise were defined as intrauterine fetal death (IUFD). Perinatal death was defined as IUFD, demise during delivery or demise in the first 7 days after delivery. Neonatal and paediatric information included GA at delivery, birth weight and sex.

\section{Statistical Analysis}

The outcome of the three groups (triplets reduced to singletons, ongoing triplets and primary singletons) was compared in terms of GA at delivery, delivery $<24$ weeks, delivery $<32$ weeks, neonatal birth weight and number of perinatal deaths.

We constructed a Kaplan-Meier curve to analyse the time to delivery in each group. As GA at delivery and neonatal birth weight were not distributed normally, we compared the median between 
Table 1. Baseline characteristics

\begin{tabular}{lcccc}
\hline & $\begin{array}{l}\text { DCTA triplet to } \\
\text { singleton }(\mathrm{n}=46)\end{array}$ & $\begin{array}{l}\text { DCTA triplet } \\
\text { ongoing }(\mathrm{n}=42)\end{array}$ & $\begin{array}{l}\text { Primary singleton } \\
(\mathrm{n}=611)\end{array}$ & p value \\
\hline $\begin{array}{l}\text { Maternal age, years } \\
\text { Nulliparity }\end{array}$ & $33.8(30.3-36.1)$ & $32.5(28.7-35.5)$ & $36.8(32.8-39.7)$ & $<0.001$ \\
Spontaneous conception & $21(45.7 \%)$ & $9(21.4 \%)$ & $398(65.1 \%)$ & $<0.001$ \\
\hline
\end{tabular}

Data are presented as median (IQR) or $\mathrm{n}(\%)$.

DCTA $=$ Dichorionic triamniotic .

the groups with the Kruskal-Wallis test. A $\chi^{2}$ test was used to compare the number of deliveries $<24$ and $<32$ weeks as well as the number of perinatal deaths among the three study groups. For perinatal mortality, we compared the absolute number of fetal deaths, the number of women who had at least one surviving child, the number of women who had at least one perinatal death and the number of women who had all their children or no children surviving. Statistical analysis was performed using Statistical Package for Social Sciences version 20.

Finally, we searched MEDLINE (November 2014) for studies analysing the outcome of fetal reduction in women with a dichorionic triplet pregnancy in which the monochorionic twin was reduced to obtain a singleton pregnancy. We included all studies that were specified for chorionicity and that reported on pregnancy outcome in terms of GA at delivery or PTB rates.

\section{Results}

We identified 46 women with a dichorionic triplet pregnancy that underwent reduction to a singleton pregnancy in the study period. During the study period, reductions were performed in 9 hospitals by 18 different operators, performing between 1 and 5 reductions each. One hospital had no reduction of a triplet to a singleton in the study period. Reasons for reduction were elective (35), TTTS (5) and congenital anomaly in 1 or 2 fetuses (3), while in 3 cases the reason for reduction could not be traced. All procedures were performed between $9^{5 / 7}$ and $17^{0 / 7}$ weeks' gestation. In 31 women the amount of potassium chloride used was mentioned. This varied between 0.8 and $12 \mathrm{ml}$, with a mean of $2.9 \mathrm{ml}$. Baseline characteristics are shown in table 1.

Figure 1 shows a Kaplan-Meier curve for time to delivery in all study groups. For women with a dichorionic reduced triplet, median GA at delivery was 38.7 weeks (IQR 35.2-40.3 weeks), compared to 32.8 weeks (IQR 29.6-34.3 weeks) for women with an ongoing dichorionic triplet pregnancy $(\mathrm{p}<0.001)$ and 40.1 weeks

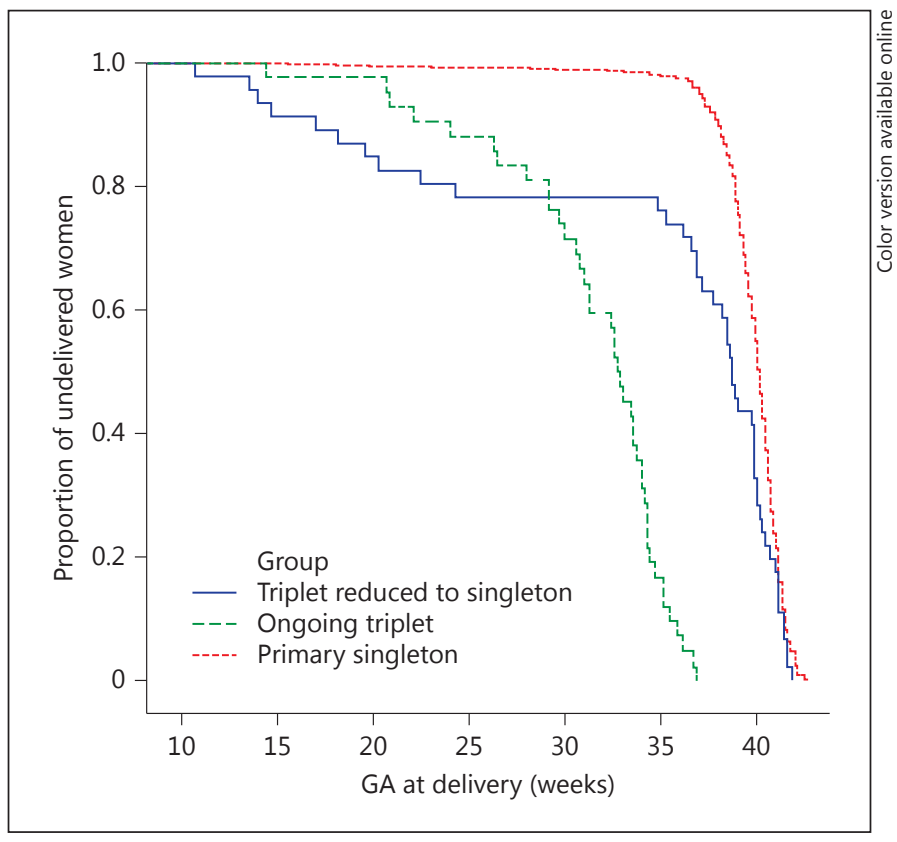

Fig. 1. Kaplan-Meier curve of time to delivery for each group.

(IQR 39.1-40.9 weeks) in women with a primary low risk singleton pregnancy $(\mathrm{p}=0.01)$ (table 2$)$. Among the women who had a reduced triplet pregnancy, 9 (19.6\%) delivered before 24 weeks' gestation compared to 4 (9.5\%) with an ongoing triplet pregnancy (RR 1.4, 95\% CI $0.91-2.2, \mathrm{p}=0.19)$ and $4(0.7 \%)$ with a singleton pregnancy delivered before 24 weeks (RR 12, 95\% CI 7.5-19, $\mathrm{p}<0.001$ ).

For delivery $<32$ weeks this was $21.7 \%$ in the reduction group vs. $40.5 \%$ in the ongoing triplet group, respectively (RR 0.63, 95\% CI 0.37-1.07, $\mathrm{p}=0.06)$ and $6(1.0 \%)$ in the singleton group (RR 11, 95\% CI 6.8-18, $\mathrm{p}<0.001$ ). Thirty women $(65.2 \%)$ with a reduced pregnancy delivered 
Table 2. Pregnancy outcome

\begin{tabular}{|c|c|c|c|c|c|}
\hline & $\begin{array}{l}\text { DCTA triplet to } \\
\text { singleton }(n=46)\end{array}$ & $\begin{array}{l}\text { DCTA triplet } \\
\text { ongoing }(n=42)\end{array}$ & $\mathrm{p}$ value $\mathrm{a}^{\mathrm{a}}$ & $\begin{array}{l}\text { Primary singleton } \\
(\mathrm{n}=611)\end{array}$ & $\mathrm{p}$ value $\mathrm{e}^{\mathrm{b}}$ \\
\hline GA at delivery, weeks & $38.7(35.2-40.3)$ & $32.8(29.6-34.3)$ & $<0.001$ & $40.1(39.1-40.9)$ & $<0.01$ \\
\hline Delivery $<32$ weeks & $10(21.7 \%)$ & $17(40.5 \%)$ & 0.06 & $6(1.0 \%)$ & $<0.001$ \\
\hline All surviving & $37(80.4 \%)$ & $30(71.4 \%)$ & 0.32 & $607(99.3 \%)$ & $<0.001$ \\
\hline None surviving & $9(19.6 \%)$ & $6(14.3 \%)$ & 0.51 & $4(0.7 \%)$ & $<0.001$ \\
\hline At least one died & $9(19.6 \%)$ & $12(28.6 \%)$ & 0.32 & $4(0.7 \%)$ & $<0.001$ \\
\hline Birth weight, g & $2,980(2,531-3,490)$ & $1,705(1,254-2,165)$ & $<0.001$ & $3,450(3,115-3,735)$ & $<0.001$ \\
\hline
\end{tabular}

Data are presented as median (IQR) or $\mathrm{n}(\%)$.

DCTA $=$ Dichorionic triamniotic.

${ }^{a} \mathrm{p}$ value for DCTA triplet to singleton vs. DCTA triplet ongoing. ${ }^{\mathrm{b}} \mathrm{p}$ value for DCTA triplet to singleton vs. primary singleton.

Table 3. Review of the literature

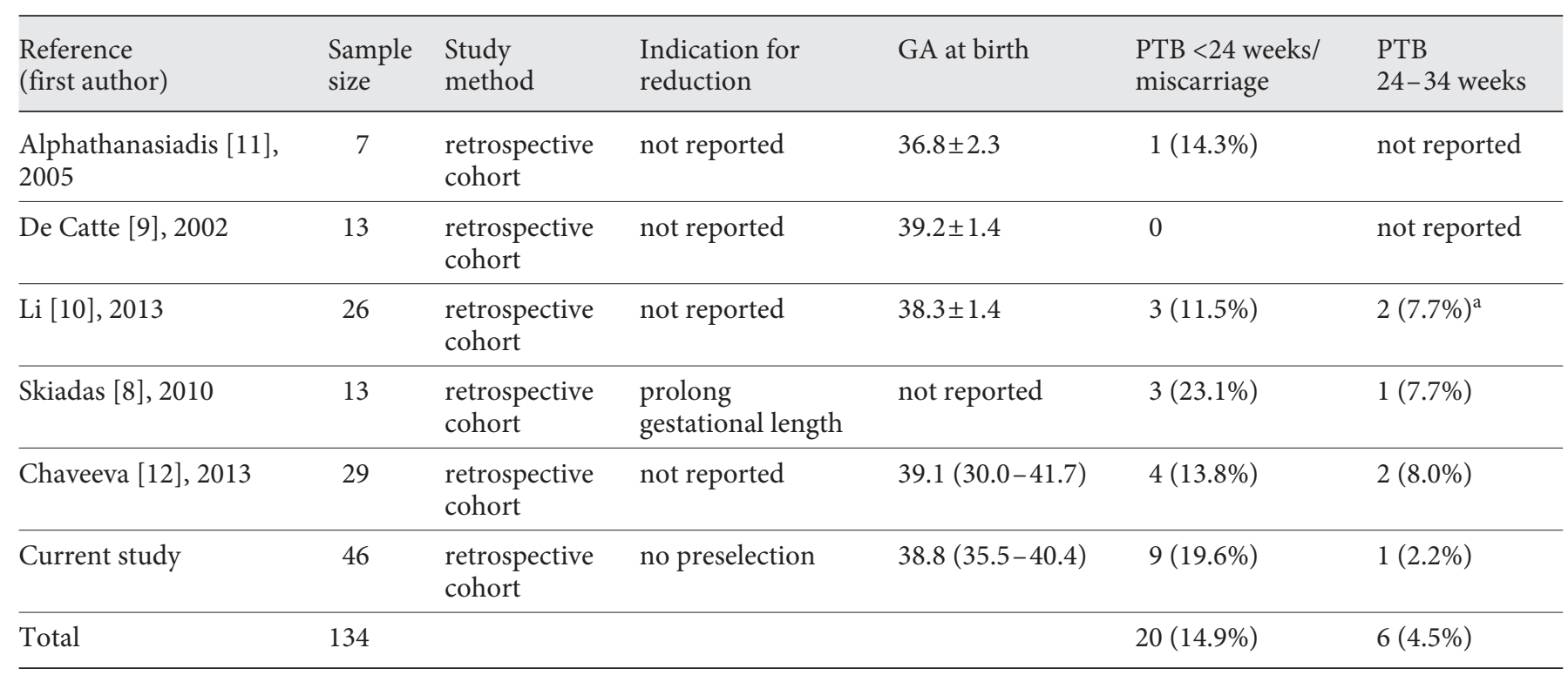

Data are presented as $\mathrm{n}(\%)$, mean $\pm \mathrm{SD}$ or median (IQR).

${ }^{\text {a }}$ PTB $<37$ week.

beyond a GA of 37 weeks. The proportion of women who had all infants surviving did not differ statistically significantly between women who had their triplets reduced to a singleton or women who had an ongoing triplet pregnancy ( 80.4 vs. $71.4 \%$, respectively, RR 0.78 , 95\% CI $0.45-$ $1.3, \mathrm{p}=0.32)$. The same holds for women who had no surviving infants (19.6 vs. $14.3 \%$, respectively, RR 1.2 , $95 \%$ CI 0.74-1.9, $\mathrm{p}=0.51)$. Birth weight was different for all groups (table 2).

Multifetal Pregnancy Reduction in Dichorionic Triplet Pregnancy
We performed a subgroup analysis for miscarriage and PTB by time of reduction and found no difference in early (before a GA of 13 weeks) and later reduction (13-17 weeks). These results are shown in online supplementary appendices 1 and 2 (see www. karger.com/doi/10.1159/000441650).

We identified five other studies [8-12] reporting on the reduction of dichorionic triplet pregnancies to a singleton pregnancy by reducing the monochorionic pair. 
These five studies reported retrospectively on a total of 88 cases (range 7-29 per study) (table 3). Mean or median GA at delivery after reduction varied from 36.8 to 39.2 weeks. All studies reported the rate of miscarriage $<24$ weeks, which varied between 0 and $23 \%$. Two studies [8, 12] reported the PTB rate $<34$ weeks as being 7.7 and $8 \%$, and one study [10] reported a PTB rate $<37$ weeks of $7.7 \%$.

\section{Discussion}

We studied the outcome of MFPR in women with a triplet pregnancy that was reduced to a singleton pregnancy. Reduction resulted in a significantly higher median GA at delivery compared to women with an ongoing dichorionic triplet pregnancy (38.7 vs. 32.8 weeks) and a non-significant higher rate of perinatal survival, but with a higher risk of delivery $<24$ weeks ( 19.6 vs. $9.5 \%$, respectively); however, this difference was not statistically significant.

Compared to those of previous studies, our results show a comparable GA at delivery, as it is reported between 36.8 and 39.1 weeks [8-12]. However the reported rates of delivery $<24$ weeks are heterogeneous between the studies, varying from 0 to $23.1 \%$, probably due to small sample sizes $(n=7-29)$. The same holds for PTB which is defined differently in all studies, varying between delivery $<32$ to $<37$ weeks. In contrast to other studies we had only one woman with a reduced triplet pregnancy who delivered between 24 and 34 weeks; this woman had an elective reduction at a GA of $11^{6 / 7}$ weeks and had a spontaneous PTB at $24^{2 / 7}$ weeks' gestation, with a surviving infant. This lower risk of (very) PTB after MFPR should be discussed with parents as prematurity and its associated complications are important contributors to long-term neonatal morbidity.

Previous studies showed that MFPR from a triplet to a singleton resulted in a trend toward, but non-significant higher rate of, delivery $<24$ weeks as compared to reduction from triplet to twin $[7,10,12]$, probably as a result of more non-vital feto-placental tissue that remains in the uterus. In this light it seems reasonable to perform reduction to twins in case of trichorionic triplets. However, in dichorionic triplets reduction to twins gives two non-favourable options: first to terminate the singleton and keep the monochorionic diamniotic twin with risks on TTTS, TAPS, selective intrauterine growth restriction and increased risk of adverse neonatal outcome [13], or second to reduce one of the monochorionic twin pair by occlusion of the umbilical cord and keep a dichorionic twin pregnancy. In a cohort of 131 women with a monochorionic twin pregnancy who underwent selective feticide of one twin, this was associated with a high risk (41.2\%) of adverse outcome for the remaining co-twin [14].

After spontaneous IUFD of one twin in a monochorionic pair there is the risk of IUFD or neurological damage to the co-twin $[7,15-17]$. Theoretically this risk is lower after selective termination of one twin than in spontaneous IUFD, but the evidence is limited to three small studies $[16,18-20]$. In case parents prefer MFPR in a dichorionic triplet pregnancy, we recommend to reduce to a singleton pregnancy by reducing both twins of the monochorionic pair. Although reduction to a singleton can result in the serious complication of losing the whole pregnancy prior to 24 weeks' gestation, the probability of delivering at term is very high.

As far as we know, this is the largest study on MFPR in women with a dichorionic triplet pregnancy to a singleton pregnancy. However, this study has some limitations. Although this is the larges study on this subject, numbers are still small and therefore the study is probably underpowered to detect differences in the amount of miscarriages and perinatal survival. The retrospective character of the study implicates that we probably missed information about women who underwent reduction. We studied all indications for reduction, whereas it would be more reasonable to differentiate between elective procedures and procedures because of obstetric or fetal indications. However, in the present study the numbers were too small to do so. Baseline characteristics were different for reduced and non-reduced triplets, obviously due to the fact that this was not a randomised trial. This could implicate selection bias, but is a reflection of daily clinical practice as well since women pregnant after ART probably make other choices than women who conceived spontaneously. In the reviewed studies there was only one [8] that defined the indication for reduction, which was to increase GA. Furthermore, we were not able to study long-term neonatal outcome, which is obviously more important than survival rates only. Moreover, we do not have reliable information about the incidence of TTTS in our control group of non-reduced dichorionic triplet pregnancies. In a previous systematic review of long-term neonatal outcome after fetal therapy including MFPR in monochorionic twins, no conclusions could be made because of limited data [16].

Restricting the number of embryos transferred in ART is the best way to prevent a high amount of multiple pregnancies and all related complications. However, triplets 
and higher-order multiples will always arise, both after spontaneous conception as well as after ART. Especially multiples with a monochorionic component can develop after natural conception [9]. To be able to counsel parents more accurately about the results and complications of MFPR, we recommend a prospective study with longterm neonatal follow-up.

\section{Disclosure Statement}

None of the authors have any conflicts of interest. No funding was received.

\section{References}

-1 Kupka MS, Ferrareti AP, de Mouzon J, Erb K, D’Hooghe T, Castilla JA, Calhaz-Jorge C, de Greyter C, Goossens V: Assisted reproductive technology in Europe, 2010: results generated from European registers by ESHRE. Hum Reprod 2014;29:2099-2113.

-2 Abusheika N, Salha O, Sharma V, Brindsen P: Monozygotic twinning and IVF/ICSI treatment: a report of 11 cases and review of literature. Hum Reprod Update 2000;6:396-403.

$\checkmark 3$ Tarlatzis C: Increase in the monozygotic twinning rate after intracytoplasmic sperm injection and blastocyst stage embryo transfer. Fertil Steril 2002;77:196-198.

4 Hall JG: Twinning. Lancet 2003;362:735-743.

$\checkmark 5$ van de Mheen L, Everwijn SMP, Knapen MFC, Oepkes D, Engels M, Manten GTR, Zondervan $\mathrm{H}$, Wirjosoekarto SAM, van Vught JMG, Erwich JJHM, Nij Bijvank SWA, Ravelli A, Heemelaar S, van Pampus MG, de Groot CJM, Mol BWJ, Pajkrt E: The effectiveness of multifetal pregnancy reduction in trichorionic triplet gestation. Am J Obstet Gynecol 2014;211:536.e1-6.

6 Lopriore E, Middeldorp JM, Oepkes D, Kanhai $\mathrm{HH}$, Walter FJ, Vandenbussche FPHA: Twin anemia-polycythemia sequence in two monochorionic twin pairs without oligopolyhydramnios sequence. Placenta $2007 ; 28$ : 47-51.

$>7$ Wimalasundera R: Selective reduction and termination of multiple pregnancies. Semin Fetal Neonatal Med 2010;15:327-335.
8 Skiadas CC, Missmer SA, Benson CB, Acker D, Racowsky C: Impact of selective reduction of the monochorionic pair in in vitro fertilization triplet pregnancies on gestational length. Fertil Steril 2010;94:2930-2931.

$\checkmark 9$ De Catte L, Camus M, Foulon W: Monochorionic high-order multiple pregnancies and multifetal pregnancy reduction. Obstet Gynecol 2002;100:561-566.

10 Li R, Chen X, Yang S, Yang R, Ma C, Liu P, Qiao J: Retain singleton or twins? Multifetal pregnancy reduction strategies in triplet pregnancies with monochorionic twins. Eur J Obstet Gynecol Reprod Biol 2013;167:146-148.

11 Alphathanasiadis AP, Zafrakas M, Tarlatzis BC, Vaitsi V, Mikos T, Bontis J: Multifetal pregnancy reduction in pregnancies with a monochorionic component. Fertil Steril 2005;83:474-476.

12 Chaveeva P, Kosinski P, Puglia D, Poon LC, Nicolaides KH: Trichorionic and dichorionic triplet pregnancies at 10-14 weeks: outcome after embryo reduction compared to expectant management. Fetal Diagn Ther 2013;34: 199-205.

13 Hack KEA, Derks JB, Elias SG, Franx A, Roos EJ, Voerman SK, Bode CL, Koopman-Esseboom C, Visser GHA: Increased perinatal mortality and morbidity in monochorionic versus dichorionic twin pregnancies: clinical implications of a large Dutch cohort study. BJOG 2008; 115:58-67.
14 van den Bos EM, van Klink JMM, Middeldorp JM, Klumper FJ, Oepkes D, Lopriore E: Perinatal outcome after selective feticide in monochorionic twin pregnancies. Ultrasound Obstet Gynecol 2013;41:653-658.

15 Ong SSC, Zamora J, Khan KS, Kilby MD: Prognosis for the co-twin following singletwin death: a systematic review. BJOG 2006; 113:992-998.

16 van Klink JMM, Koopman HM, Oepkes D, Walther FJ, Lopriore E: Long-term neurodevelopmental outcome in monochorionic twins after fetal therapy. Early Hum Dev 2011; 87:601-606.

17 Shek NW, Hillman SC, Kilby MD: Singletwin demise: pregnancy outcome. Best Pract Res Clin Obstet Gynaecol 2014;28:249-263.

-18 Lewi L, Gratacos E, Ortibus E, van Schoubroeck D, Carreras E, Higueras T, Perapoch J, Deprest J: Pregnancy and infant outcome of 80 consecutive cord coagulations in complicated monochorionic multiple pregnancies. Am J Obstet Gynecol 2006;194:782-789.

19 Moise KJ Jr, Johnson A, Moise KY, Moise RN, Nickeleit V: Radiofrequency ablation for selective reduction in the complicated monochorionic gestation. Am J Obstet Gynecol 2008;198:198.e1-5.

20 Robyr R, Yamamoto M, Ville Y: Selective feticide in complicated monochorionic twin pregnancies using ultrasound-guided bipolar cord coagulation. BJOG 2005;112:1344-1348. 